\title{
Evaluación de las Ramas Diagonales, Anterosuperior, Posterolateral Derecha y Lateral del Corazón. Un Estudio en Individuos Colombianos
}

\author{
Evaluation of the Heart Diagonal, Anterosuperior, Right Posterolateral \\ and Lateral Branches. A Study in Colombian Individuals
}

"Luis Ernesto Ballesteros; * Luis Miguel Ramirez \& ${ }^{* * *}$ Vladimir Saldarriaga

BALLESTEROS, L. E.; RAMIREZ, L. M. \& SALDARRIAGA, V. Evaluación de las ramas diagonales, anterosuperior, posterolateral derecha y lateral del corazón. Un estudio en individuos colombianos. Int. J. Morphol., 27(4):1073-1080, 2009.

RESUMEN: Las arterias que irrigan la cara pulmonar del corazón y la superficie posterior del ventrículo derecho presentan una expresión variable. Se evaluaron 154 corazones frescos de material de autopsia. Las arterias coronarias fueron sometidas a proceso de inyección con resinas sintéticas. Se observó la rama posterolateral derecha (sólo en dominancia derecha) en 24 corazones (15,6 \%), de los cuales $20(83,3 \%)$ se originaron directamente de la arteria coronaria derecha y 4 de la marginal derecha. Ésta estuvo presente en el $40 \%$ de los casos que tenían una rama interventricular posterior corta y en el 5,6\% con presencia de rama larga ( $\mathrm{p}=0,001)$. Se observó además, ramas diagonales en 74 corazones $(48 \%)$, ramas anterosuperiores en $126(81,8 \%)$ y ramas laterales en $123(79,9 \%)$. Las ramas de longitud media fueron las más frecuentes: diagonales $(52,3 \%)$; anterosuperiores $(62,7 \%)$ y laterales $(47,1 \%)$. El calibre de las ramas diagonales fue de 2,01 mm (DE 0,59). De las ramas diagonales, las que presentaron menor calibre correspondieron a los especímenes con dominancia izquierda 1,88 mm DS 0,52), sin ser significativo (P 0,608), mientras que las ramas laterales de mayor calibre correspondieron a corazones con dominancia izquierda $(\mathrm{p}=0,022)$. De las ramas diagonales se observaron puentes miocárdicos en 11 muestras $(14,9 \%) ; 6(4,8 \%)$ en las ramas anterosuperiores y $2(8,3 \%)$ en los corazones que presentaban ramas posterolaterales derechas. Las ramas laterales no presentaron puentes miocárdicos. Se observó presencia de la rama posterolateral derecha en un sexto de los individuos con dominancia derecha y las ramas diagonales presentaron, en su mayoría, un curso epicárdico que finalizó en el tercio medio de la cara obtusa del corazón. coronaria.

PALABRAS CLAVE: Rama diagonal; Rama anterosuperior; Rama posterolateral derecha; Rama lateral; Circulación

\section{INTRODUCCIÓN}

La rama posterolateral derecha (RPLD) se desprende de la arteria coronaria derecha (ACD) cerca del margen agudo del corazón y de manera ocasional de la rama marginal derecha (RMD). Adopta una trayectoria oblicua sobre la pared posterior del ventrículo derecho, alcanzando el tercio intermedio del surco interventricular posterior (SIP) (Sewell, 1966). Ha sido denominada por Nerantzis (Nerantzis et al., 1994) como arteria diagonal derecha posterior y como el tronco menor de la división de la arteria coronaria derecha por James (James, 1965), que se deriva a nivel del margen agudo. Otros autores la han denominado rama lateral (Campbell, 1929); reflexión posterior de la RMD (Smith,
1962) y segunda arteria descendente posterior (McAlpin, 1975). Esta arteria es de carácter inconstante y se presenta en los corazones con dominancia derecha, en un rango del $14 \mathrm{al} 20 \%$ y está fuertemente asociada con la presencia de ramas interventriculares posteriores (RIP) cortas, que terminan en el segmento superior o intermedio del SIP, contribuyendo de esta forma con la irrigación del segmento inferior de la cara diagframática del corazón (Nerantzis et al., 1994; Margaris et al., 1997, Ortale et al., 2004)

En relación a la rama diagonal, se presenta confusión entre los diferentes autores para denominarla. Unos la

\footnotetext{
Profesor Titular. Departamento Ciencias Básicas. Universidad Industrial de Santander. Bucaramanga, Colombia. Profesor Universidad Autónoma de Bucaramanga, Colombia.

** Profesor Asociado. Director Investigaciones Postgrado. Universidad Santo Tomas de Aquino. Bucaramanga, Colombia.

*** Profesor Asociado. Coordinador Línea Ciencias Básicas. Universidad Autónoma de Bucaramanga, Colombia.
} 
denominan como originada del tronco de la ACI formando una bisectriz que surge entre la RIA y la RCX (Baptista $e t$ al., 1991; Reig \& Petite, 2004). Otros autores utilizan una denominación más amplia considerando a esta arteria originada también en el vértice del ángulo formado por las ramas terminales de la ACI, pero contemplando igualmente su emergencia de los milímetros iniciales de la RIA o la RCX (James, 1961; Angelini et al., 1999). La irrigación de la cara obtusa del corazón, especialmente de sus segmentos superior e intermedio, puede darse además de las RDs, por la rama anterosuperior (RAS) la cual se desprende del segmento proximal de la RIA y por la rama lateral (RL) desprendida de la RCX. Estas ramas presentan variabilidad relacionada con su presencia o ausencia, calibres y longitud de su curso subepicárdico (Baptista et al., 1991; Didio \& Wakefield, 1975; Ortale et al., 2005). Características que permiten interpretar los diversos eventos clínicos y realizar las correspondientes maniobras intervencionistas.

Los puentes miocárdicos (PM) considerados como segmentos sumergidos de las arterias coronarias o de sus ramas en el miocardio, presentan una frecuencia variable, la cual depende de la naturaleza del estudio, métodos de registro, grupos poblacionales y género. En estudios angiográficos han sido reportados en un rango del 0,5 al 12\% (Çay et al., 2006; Kantarci et al., 2006; Ko et al., 2008), mientras que en trabajos anatómicos se ha informado con frecuencias de 5,4 al 85,7\% (Polacek, 1961; Bezerra et al., 1987; Loukas et al., 2006). La estructura arterial que presenta mayor frecuencia de PM es la rama interventricular anterior (RIA) (12\%63\%). En las RD, RPLD, RAS y RL, la presencia de PM es baja (3-7\%) (Sahni \& Jit, 1991; Baptista \& DiDio,1992; Kosinski \& Grzybiak, 2001; Loukas et al.).

Los PM considerados por unos como variaciones anatómicas y por otros como malformaciones vasculares, están involucrados en eventos patológicos como arritmias, infarto de miocardio o incluso muerte súbita (Quagliano et al., 1992; Gow, 2002; Rozemberg \& Nepomnyashchikh, 2004).

El conocimiento de los diversos patrones de expresión morfológica de las arterias coronarias y de sus ramas derivadas, se hace de vital importancia para adecuadas interpretaciones y aplicaciones especialmente por su evidente variabilidad. En este sentido, la determinación de territorios relacionados con la enfermedad coronaria oclusiva e igualmente los procedimientos imagenológicos, hemodinámicos intervencionistas y quirúrgicos se fundamentan en este conocimiento.

Las RD, RL, y RPLD han sido descritas en otros grupos poblacionales, pero en colombianos no hay información. Consecuentemente, este estudio pretende determinar la frecuencia y expresión morfológica de estas arterias origina- das de las coronarias derechas e izquierdas, en una muestra de material cadavérico fresco de población colombiana; evaluando además la correlación del calibre de estos vasos con el tipo de dominancia coronaria.

\section{MATERIAL Y MÉTODO}

Se evaluaron los corazones obtenidos de 154 cadáveres frescos (125 hombres; 29 mujeres), a quienes se les practicó necropsia en el Instituto de Medicina Legal de Bucaramanga (Colombia). La muestra cumplió con los siguientes criterios de inclusión: sujetos masculinos y femeninos del grupo racial mestizo, sin signos de patología o trauma cardiaco.

Mediante canalización de su ostium, las arterias coronarias fueron sometidas a un proceso de inyección con resina sintética (palatal GP41L 80\% y estireno 20\%) a una presión de $120 \mathrm{~mm}$ de $\mathrm{Hg}$, luego los corazones se dejaron en solución de $\mathrm{KOH}$ al $15 \%$ por 5 minutos para liberar la grasa epicárdica (Nerantzis, et al., 1978). Se determinó la dominancia coronaria con los criterios de Schlesinger (Schlesinger, 1940). Igualmente, la presencia de la RPLD y su origen de la ACD o de la RMD. Se consideró a la RIP como larga cuando finalizaba en el tercio inferior del SIP o en el ápex; y corta cuando finalizaba en los segmentos superiores y medio del SIP. Se determinó la presencia de RD cuando ésta emergía como una bisectriz de la división de la ACI en sus RCX y RIA. Se consideró como RAS a las ramas derivadas de la parte más proximal de la RIA; y RL a las ramas derivadas de la parte más proximal de la RCX (Ortale et al., 2005). Se registró el calibre de estas ramas y principales colaterales a $5 \mathrm{~mm}$ de sus orígenes, con calibrador electrónico Mitutoyo. Adicionalmente, se determinó la longitud del ventrículo izquierdo desde el punto donde la ACI se divide hasta el ápex del corazón. Las RD, RAS y RL fueron clasificadas como cortas, medias o largas si la distancia entre su origen y el punto de penetración dentro del miocardio alcanzaba los tercios superior, medio e inferior de la longitud ventricular, respectivamente. Se correlacionó el calibre de las RD, RAS y RL con la dominancia coronaria. Se registraron la frecuencia, longitud y profundidad de los PM presentes. De cada una de las piezas evaluadas se obtuvieron registros fotográficos.

En el análisis de los datos, las variables continuas fueron descritas con sus promedios y desviación estándar, igualmente, las variables nominales con sus proporciones. Se realizaron las pruebas estadísticas de chi $\left(\mathrm{X}^{2}\right)$ cuadrado aceptando un error alfa hasta de un 5\%. La base de datos se digitó en Excel y los análisis estadísticos se realizaron en STATA 8.0. 


\section{RESULTADOS}

La edad promedio de los individuos en que se obtuvieron las muestras, fue de 31.4 años (16-75 años) y el peso promedio fue de 291,2g DS 45,5 (hombres 300,7 DS 58; mujeres 248,4 DS 61,1).

En $129(83,7 \%)$ de los especímenes cardiacos se observó dominancia coronaria derecha. La circulación balanceada en $14(9,2 \%)$; y la dominancia izquierda en nueve corazones $(7,2 \%)$. La dominancia derecha se presentó con mayor frecuencia en mujeres, pero no fue estadísticamente significativa $(\mathrm{p}=0,26)$.

Se observó RPLD en 24 corazones (hombres 20 $(16 \%)$, mujeres $4(13,8 \%)$ ) en un $15,6 \%$ y solamente en los especímenes con dominancia derecha $(18,6 \%)$ de los cuales $20(83,3 \%)$ se originaron de la ACD y $4(17,7 \%)$ de la marginal derecha. La distancia entre el origen de las RPLD y el de las RMD fue de 13,4 mm DS 5,5. Las RPLD con un

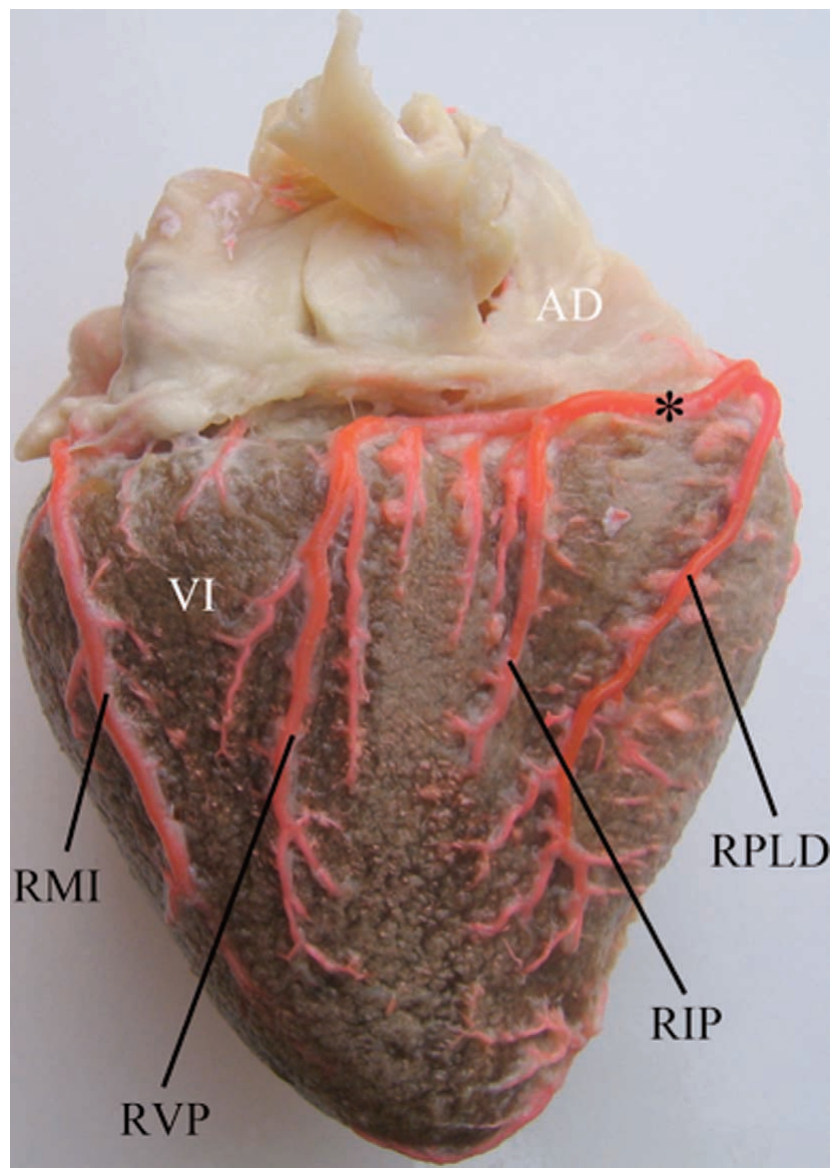

Fig. 1. Cara diafragmática del corazón. AD. Atrio derecho; VI. Ventrículo izquierdo; RPLD. Rama posterolateral derecha; RIP. Rama interventricular posterior (corta); RVP. Rama ventricular posterior; RMI. Rama marginal izquierda. calibre de $1,9 \mathrm{~mm}$ DS 0,48, se desprendieron de la ACD, la cual presentaba, a ese nivel, un calibre de 2,72 mm DS 0.4. La longitud de su primer segmento, con una trayectoria oblicua sobre la cara posterior del ventrículo derecho (hasta alcanzar el SIP) fue de $53,7 \mathrm{~mm}$ DS 7,75. Su curso a lo largo del SIP fue de 20,9 mm DS 6,2, y su finalización se dio a una distancia de 19,5 mm DS 6,1 del ápex cardiaco.

La expresión larga de la RIP alcanzó el tercio inferior del surco homónimo y el ápex, en 108 de los especímenes $(70,6 \%)$ y fue corta cuando finalizó en los segmentos superior y medio del surco en 45 corazones $(29,4 \%)$. De los casos con RIP corta, se observó la presencia de RPLD en el $40 \%$ (12 muestras con la RIP terminando en el tercio superior y 6 casos en el tercio medio del SIP) (Fig. 1). En las muestras con RIP largas, se observó concomitantemente la presencia de RPLD en el 5,6\% ( $\mathrm{p}=0,001)$.

La longitud del ventrículo izquierdo fue de $93,7 \mathrm{~mm}$ DS 7,6. La ACI originó una RD única en 65 muestras (42\%) y dos RD en 9 (5,8\%). Las RD presentaron en la forma trifurcada una longitud de $56 \mathrm{~mm}$ DS 21,6 y un calibre de 2,01 mm DS 0,59. En los casos de tetrafurcación, la longitud observada fue de $37,7 \mathrm{~mm}$ DS 14,9 con calibre de 1,54 mm DS 0,45. Las RAS fueron observadas en 126 especímenes $(81,8 \%)$, con una la longitud de 50,2 $\mathrm{mm}$ DS $16.1 \mathrm{y}$ un calibre de $1,69 \mathrm{~mm}$ DS 0,43 , mientras que 123 $(79,9 \%)$ de los corazones presentaron las RL con una longitud de 33,4 mm DS 17,8 y el calibre de 1,22 mm DS 0,33.

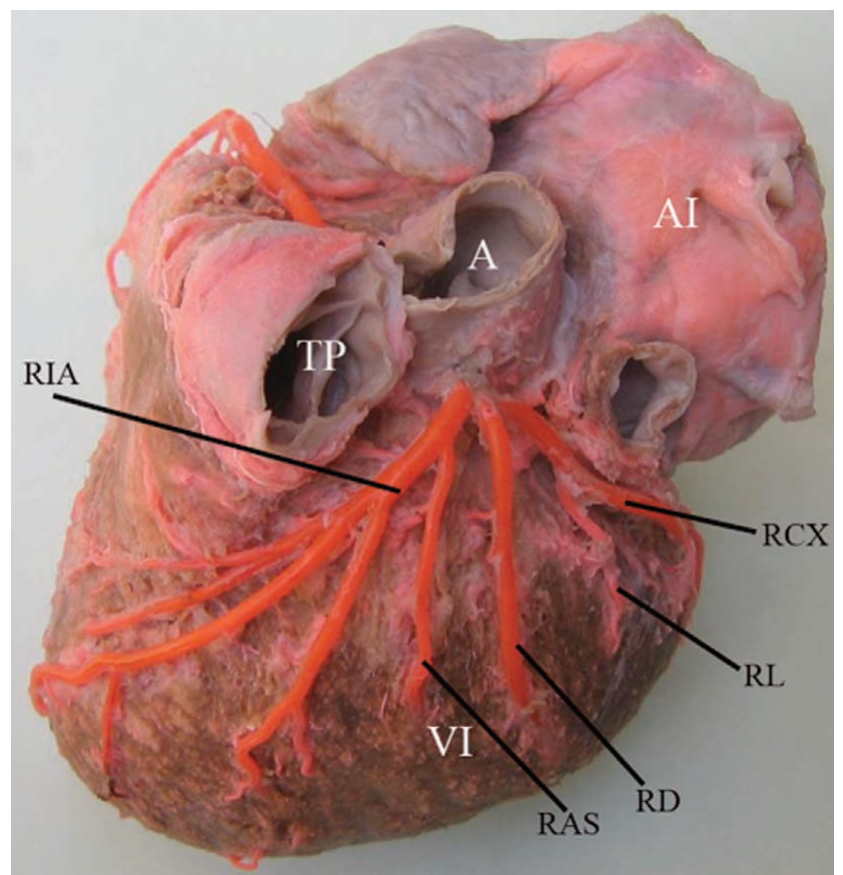

Fig. 2. Cara anterior del corazón. AI. Atrio izquierdo; A. Aorta; TP. Tronco pulmonar; RCX. Rama circunfleja; RL. Rama lateral; RD. Rama diagonal; RAS. Rama anterosuperior; RIA. Rama interventricular anterior. 
BALLESTEROS, L. E.; RAMIREZ, L. M. \& SALDARRIAGA, V. Evaluación de las ramas diagonales, anterosuperior, posterolateral derecha y lateral del corazón. Un estudio en individuos colombianos. Int. J. Morphol., 27(4): 1073-1080, 2009.

Tabla I. Frecuencia de los tipos de las ramas diagonal, anterosuperior y lateral en 154 corazones de individuos colombianos de ambos sexos.

\begin{tabular}{|c|c|c|c|c|c|c|c|c|}
\hline & \multicolumn{2}{|c|}{ Cortas } & \multicolumn{2}{|c|}{ Medias } & \multicolumn{2}{|c|}{ Largas } & \multicolumn{2}{|c|}{ Total } \\
\hline & $\mathrm{N}$ & $\%$ & $\mathrm{~N}$ & $\%$ & $\mathrm{~N}$ & $\%$ & $\bar{N}$ & $\%$ \\
\hline RD1 & 5 & 7,7 & 34 & 52,3 & 26 & 40,0 & 65 & 100 \\
\hline Hombres & 3 & 5,7 & 27 & 50,9 & 23 & 43,4 & 53 & 100 \\
\hline Mujeres & 2 & 16,7 & 7 & 58,3 & 3 & 25,0 & 12 & 100 \\
\hline $\mathrm{RD} 2$ & 3 & 2,0 & 5 & 3,2 & 1 & 0,6 & 9 & 100 \\
\hline Hombres & 3 & 37,5 & 4 & 50,0 & 1 & 12,5 & 8 & 100 \\
\hline Mujeres & 0 & 0,0 & 0 & 0,0 & 1 & 100 & 1 & 100 \\
\hline Rama anterosuperior & 17 & 13,5 & 79 & 62,7 & 30 & 23,8 & 126 & 100 \\
\hline Hombres & 15 & 14,7 & 61 & 59,8 & 26 & 25,5 & 102 & 100 \\
\hline Mujeres & 2 & 8,3 & 18 & 75,0 & 4 & 16,7 & 24 & 100 \\
\hline Rama lateral & 59 & 48,0 & 58 & 47,1 & 6 & 4,9 & 123 & 100 \\
\hline Hombres & 44 & 45,4 & 49 & 50,5 & 4 & 4,1 & 97 & 100 \\
\hline Mujeres & 15 & 57,7 & 9 & 34,6 & 2 & 7,7 & 26 & 100 \\
\hline
\end{tabular}

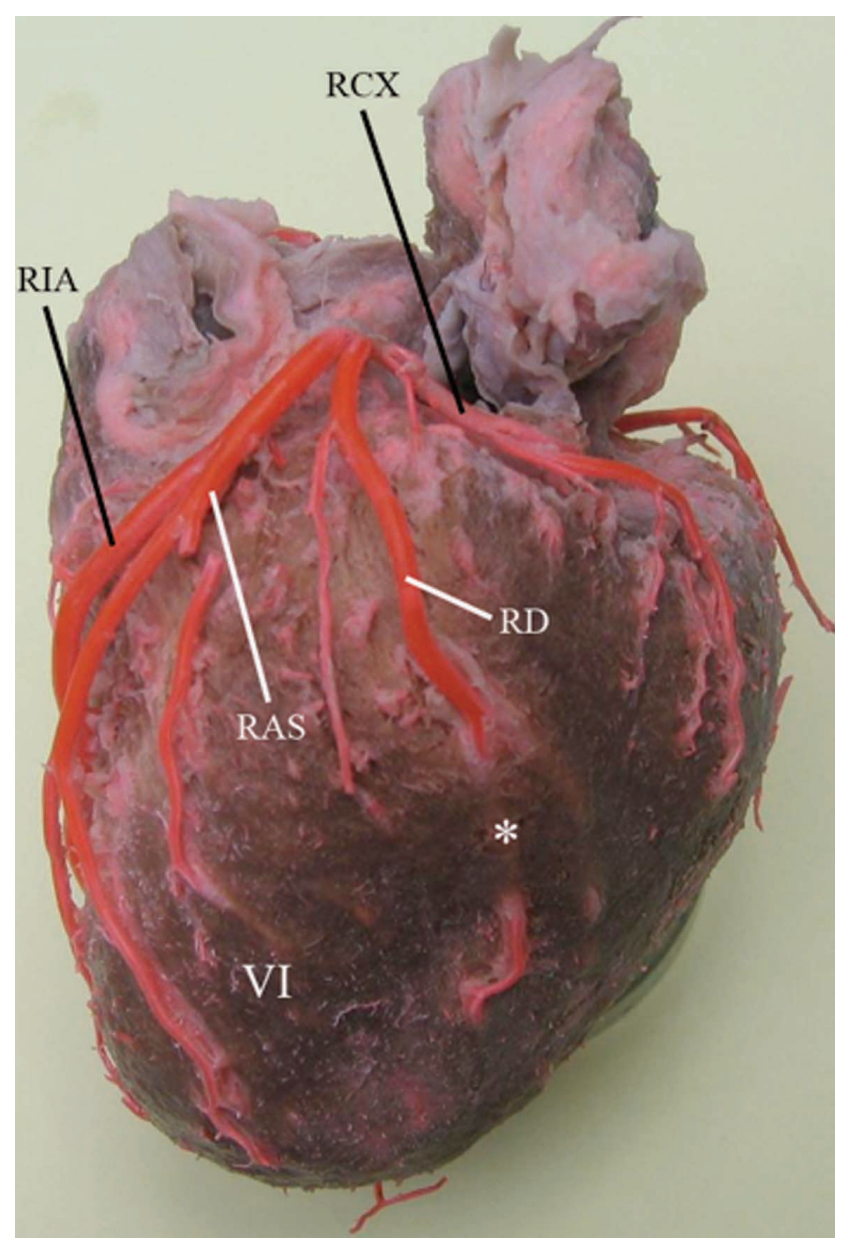

Fig. 3. Cara pulmonar del corazón. VI. Ventrículo izquierdo; RCX. Rama circunfleja; RD. Rama diagonal; RAS. Rama anterosuperior; RIA. Rama interventricular anterior; * Puente Miocárdico.
En 34 casos (52,3\%) con ACI trifurcada, se observó RD de longitud media (mujeres 58,3\% y hombres 50,9\%) (Fig. 2) y menos frecuentes fueron las RD de longitud corta. Las RAS de longitud media también fueron las más frecuentes $(62,7 \%)$ tanto en hombres como en mujeres. Mientras que en las RL fue similar la frecuencia de las cortas y medias (Tabla I).

En relación con el tipo de irrigación coronaria, las RD que presentaron menor calibre correspondieron a los especímenes con dominancia izquierda (1,88 mm DS 0,52), sin diferencia estadística $(\mathrm{p}=0,608)$, con relación a los vasos de corazones con dominancia derecha. Igualmente, las RAS de las muestras con dominancia derecha presentaron mayor calibre en relación con la dominancia izquierda ( $\mathrm{p}=$ $0,05)$; mientras que las RL de mayor calibre correspondieron a corazones con dominancia izquierda $(\mathrm{p}=0,022)(\mathrm{Ta}-$ bla II).

Se observó PM (Fig. 3) en 11(14,9\%) de las 74 RD presentes en la muestra, en $6(4,8 \%)$ de las 126 RAS y en 2 $(8,3 \%)$ de los corazones que presentaban RPLD. Las RL no presentaron PM. El espesor del miocardio suprapontino fue de $1,55 \mathrm{~mm}$ DS 0,71. Los PM localizados en las RD y RAS presentaron una longitud de $29,7 \mathrm{~mm}$ DS 14,5 y $16,2 \mathrm{~mm}$ DS 8,3 respectivamente, mientras que en los localizados en la RPLD la longitud fue de 24,9 mm (rango 7,7 - 39,4 $\mathrm{mm})$. 
Tabla II. Calibres en mm de las ramas diagonales, anterosupeior y lateral en 154 corazones de individuos colombianos en relación con el tipo de dominancia coronaria.

\begin{tabular}{|c|c|c|c|c|c|c|}
\hline & Dom & echa & Domi & lier da & Circul & ceada \\
\hline & & D.S. & & D.S. & & D.S. \\
\hline RD & 2,01 & 0,49 & 1,88 & 0,52 & 2,15 & 0,39 \\
\hline RAS & 1,72 & 0,41 & 1,27 & 0,39 & 1,6 & 0,24 \\
\hline RL & 1,2 & 0,26 & 1,71 & 0,68 & 1,08 & 0,58 \\
\hline
\end{tabular}

\section{DISCUSIÓN}

La RPLD es una estructura vascular que ha causado gran confusión en la literatura en relación con el nombre, origen, curso y finalización. En concordancia con Nerantzis et al., (1994) y Margaris et al., no observamos a ésta en corazones con dominancia izquierda. Ortale et al. (2004) la reportan en los tres tipos de circulación coronaria.

En relación a la frecuencia de prevalencia, nuestros hallazgos son similares a los reportados por Nerantzis et al., (1994) 14.5\%, Margaris et al. 15,5\% y Ortale et al. (2004) 17,5\%. Ortale et al. (2004) además encontraron esta estructura en el 2,5\% de los corazones con circulación balanceada y en el $5.5 \%$ de las piezas con circulación izquierda. Cuando no existe RPLD en el segmento entre la emergencia de la RMD y la cruz cordis, surgen de la ACD pequeñas ramas que suplen la pared posterior del ventrículo derecho. Estas ramas de pequeño calibre son difíciles de identificar en los estudios angiográficos. Igualmente, puede llegar a confundirse la RPLD con una interventricular accesoria (Margaris et al.)

La distancia de origen de la RPLD en relación con el origen de la RMD fue reportada por Nerantzis et al. (1994) en un rango de 3 a $28 \mathrm{~mm}$, el cual podría catalogarse como similar a la medida realizada en nuestro estudio $(13,4 \mathrm{~mm})$ Igualmente, existe concordancia con estos autores en torno a la trayectoria y a la distancia de la RPLD desde su origen al SIP (53,7 y $61 \mathrm{~mm})$ y a la longitud de esta arteria en su curso por este surco (20,9 y $22 \mathrm{~mm})$. En ninguna de las muestras evaluadas encontramos la RPLD finalizando en el ápex, mientras que Nerantzis et al. (1994) reportan que en el $8,1 \%$ de los casos esta arteria finaliza en el surco interventricular anterior de 7 a $15 \mathrm{~mm}$ del ápex.

El origen de la RPLD observado en nuestro estudio a partir de la ACD (83,3\%) y de la RMD (17,7\%) es concordante con los reportes de Nerantzis et al. (2004) y Margaris et al. (84 y 90\% en la ACD; 10 y $16 \%$ de RMD). El hecho que en el 10-17,7\% se origine o sea continuación de la marginal, permite entender por qué Smith la denominó como reflexión posterior de la marginal y
Campbell la denominara rama lateral. Igualmente, existe acuerdo con los anteriores autores en relación al calibre menor de la RPLD (1,2-1,9 mm) con relación al vaso que la origina, bien fuera la ACD o la RMD. En controversia con nuestros hallazgos y los de los autores anteriormente expuestos, Sewell reporta que estos vasos son de similar calibre a la ACD y es por ello que la considera una continuación de ésta y no como una de sus ramas. Igualmente, el reporte de James (1965) que considera la RPLD como si fuera el tronco inferior y menor de la división ACD y derivada a nivel del margen agudo del corazón, lo que no ha tenido acogida en razón a su alta ausencia (80-85\%) y escaso calibre.

La presencia de RIP largas $(70,6 \%)$ que alcanzan el tercio inferior del surco interventricular posterior o el ápex, son ligeramente menores a lo reportado por James (1965), Nerantzis et al. (1994) y Margaris et al. Se destaca la concordancia con estos trabajos en torno al porcentaje de RIP cortas que se acompañan de RPLD (39-40\%) y al escaso número de casos de RIP largas que presentan concomitantemente RPLD (Nerantzis et al.,1994; 8\%; Margaris et al. 6\% y en nuestro estudio 5,6\%). Estas observaciones son de importancia práctica, en razón a que la RPLD perfunde la parte inferior del surco interventricular posterior y su área adyacente junto con la RIA. Cuando ésta persiste junto a una RIP larga, contribuye a la irrigación del septum interventricular y área adyacente. Lo anterior limita en las zonas descritas, la posibilidad de procesos isquémicos. En presencia de oclusión de la RIP, la RPLD se constituye en un bypass o puente anastomótico que suple el tercio inferior de la cara diafragmática.

Efectivamente, la presencia de la RPLD es una expresión anatómica que se convierte en factor protector que evita la isquemia del sector inferior de la cara diafragmática, cuando se necesita.

Estudios previos han reportado una amplia variación en la frecuencia de ramas diagonales como producto de la división trifurcada o tetrafurcada del tronco de la 
ACI. La presencia de una rama diagonal, observada en este trabajo en el $42,2 \%$, se ubica en un rango intermedio en relación a trabajos previos que informan frecuencias de rangos entre un 9 al 60\%: Baptista \& DiDio 38,7\%, Kalbfleisch et al. (1977) 55\%; Cavalcanti et al. 38,5\%; Penthe et al. (1976) 9\%; Kalpana (2003) 40\%; (Leguerrier et al. (1976) 26\%; De Paula (1972) 60,2 y Ortale et al. (2005) 46\%. En contraste, Grande et al. (1982) quienes informaron la presencia de una RD en un $82,5 \%$.

La frecuencia de dos RD en cada corazón observada en esta serie (5,8\%), fue similar ( 5 al 7\%) a lo señalado por Leguerrier et al.; Kalbfleisch et al.; Baptista \& DiDio y Ortale et al. (2005). En concordancia con los reportes de Baptista, de Paula y Lima, en nuestro estudio predominan las RD que penetran al miocardio a nivel del tercio medio de la superficie ventricular, mientras que Ortale et al. (2005) y Banchi (1904), reportan predominio de las RD cortas. Las variaciones en el largo de las ramas diagonales tienen especial importancia en cirugía cardiaca porque su porción subepicardica es frecuentemente usada para implante de injerto bypass (Acar et al., 1993), por lo que la presencia de RD cortas limitan la posibilidad de realizar este tipo de procedimientos.

La frecuencia de las RAS y RL reportada por Ortale et al., (2005) (82 y 88\%, respectivamente) es similar a lo que se reporta en el presente trabajo. Los calibres de las RD y RAS reportados por Ortale et al., (2005) de 2,2 y $25 \mathrm{~mm}$, respectivamente, son ligeramente mayores a nuestros hallazgos; mientras que el calibre de las RL, medido en nuestro estudio, fue significativamente menor (1,22 mm) al reportado por Ortale et al., (2005) de 2,1 mm. La valoración del calibre de estos vasos cobra importancia para la realización de procedimientos de revascularización cardiaca, en razón a que para realizar estos procedimientos se requiere que los vasos tengan mínimo $1,5 \mathrm{~mm}$ de diámetro (Acar et al.).

En concordancia con el trabajo de Ortale et al., (2005), encontramos en nuestro estudio que las RD presentan mayor calibre en los corazones con circulación coronaria balanceada, mientras que el calibre de las RAS es mayor en las muestras con dominancia derecha, con relación a las que presentan dominancia izquierda. En contraste, el calibre de las RL es significativamente mayor en los especímenes con dominancia izquierda. Esto sugiere que la distribución de la irrigación de la cara pulmonar del corazón, especialmente en lo relacionado con el calibre de las diversas ramas que suplen, pudiera estar influenciado por el tipo de dominancia coronaria, lo cual se traduce en que, dependiendo del tipo de dominancia, ciertas áreas tendrían un mayor flujo sanguíneo relativo que otras, presen- tando cierta vulnerabilidad ante un incremento del gasto cardiaco.

La frecuencia de PM observada en las RD (14,9\%), es superior a la prevalencia reportada por Sahni \& Jit, Baptista \& DiDio, Kosinski \& Grzybiak. Por otro lado, se consideró similar a los hallazgos de Loukas et al. Igualmente, hay acuerdo con los anteriores autores en relación a la infrecuente localización de PM en las RAS y en ramas de la coronaria derecha como la RPLD.

Los trabajos de Loukas et al., Rozemberg \& Nepomnyashchikh y Lima et al. (2002) reportaron longitudes de los PM en un rango de 25 a $31 \mathrm{~mm}$, los cuales coinciden con nuestros hallazgos. Son reportados por Reig et al. (1986), Canyigit et al. (2009); Ko et al., menor extensión de estos túneles vasculares (13 a18 mm). La profundidad de los PM encontrados en nuestro estudio (1,54 $\mathrm{mm}$ ) se halla en un nivel intermedio entre los hallazgos de Kosinzky \& Grzybiak (1,8 mm), Kantarci et al., (2,5 mm) y Ko et al., (3 mm), y los más bajos reportados por Reig et al. (0,9 mm), Loukas et al., (1,2 mm), Canyigit et al., (1,3). La longitud, la profundidad y la ubicación en una o en varias ramas arteriales, son características morfológicas de los PM que pueden incidir en la expresión de diversas patológicas isquémicas o arrítmicas del corazón. Es importante tener en cuenta que los PM en un alto porcentaje cursan de manera asintomática, en razón a que se presentan mecanismos de compensación como la presencia de ramas colaterales que surgen en el segmento prepontino y contribuyen a la irrigación de territorios adyacentes. Igualmente, cuando se suman al substrato anatómico relativamente adverso, factores como la ateromatosis pueden desencadenar eventos clínicos como angina, arritmias o incluso la muerte súbita ante situaciones de alto gasto cardiaco como el estrés y el ejercicio (Rozemberg \& Nepomnyashchikh, Gow et al., Duygu et al., 2007).

Este trabajo de variaciones vasculares cardiacas matiza nuevamente la importancia del conocimiento de sus variaciones, por sus repercusiones en el diagnóstica y el manejo de los eventos clínicos correspondientes. Igualmente. contribuye en la finesa del diagnóstico imagenológico cuando estas variaciones están presentes.

\section{AGRADECIMIENTOS}

Al Instituto de Medicina Legal y Ciencias Forenses por la donación de las piezas requeridas para este estudio. Al señor Carlos Bermúdez, Técnico de Laboratorio, por su valiosa asistencia en la toma de registros fotográficos. 
BALLESTEROS, L. E.; RAMIREZ, L. M. \& SALDARRIAGA, V. Evaluation of the heart diagonal, anterosuperior, right posterolateral and lateral branches. A study in Colombian individuals. Int. J. Morphol., 27(4):1063-1067, 2009.

SUMMARY: The arteries that irrigate the lung face of the heart and the posterior surface of the right ventricle present a variable expression. 154 fresh hearts were evaluated obtained as autopsy material. The coronary arteries were subjected to injection process with synthetic resins. Right posterolateral branch (right dominance) was observed in 24 hearts (15.6\%), of which 20 (83.3\%) originated of the right coronary artery and 4 of the marginal right artery. Within these in $40 \%$ of the cases were observed in short posterior interventricular branch and 5.6\% with long branch $(\mathrm{p}=0.001)$. It was observed diagonal branches in 74 hearts $(48 \%)$, anterosuperior branches in 126 $(81.8 \%)$ and lateral branches in 123 (79.9\%). The branches of middle longitude were the most frequent: diagonals (52.3\%); anterosuperiors $(62.7 \%)$ and laterals $(47.1 \%)$. The calliper of the diagonal branches was of $2.01 \mathrm{~mm}$ (SD 0.59). Of the diagonal branches, those that presented smaller calliper corresponded to the specimens with left dominance (1.88 mm SD 0.52), without significance (P 0.608), while the lateral branches of more calliper corresponded to hearts with left dominance $(\mathrm{p}=0.022)$. It was also observed myocardial bridges in 11 diagonal branches (14.9\%); in 6 anterosuperior branches (4.8\%) and in 2 right posterolateral branches (8.3\%). The lateral branches do not presented myocardial bridges. Prevalence of the right posterolateral branch was observed in a sixth of the individuals with right dominance, and the majority of diagonal branches presented an epicardic course that concluded in the middle third of the obtuse face of the heart. circulation.

KEY WORDS: Diagonal branch; Anterosuperior branch; Right posterolateral branch; Lateral branch; Coronary

\section{REFERENCIAS BIBLIOGRÁFICAS}

Acar, C.; Farge, A.; Chardigny, C.; Beyssen, B.; Pagny, J. Y. Grare, P.; Fabiani, J. N.; Deloche, A.; Guermonprez, J. L. \& Carpentier, A. Use of the radial artery for coronary artery bypass. A new experience after 20 years. Arch. Mal. Coeur. Vaiss., 86:1683-9, 1993.

Angelini, P.; Villason, S.; Chan, A. V. \& Diez, J. G. Normal and anomalous coronary arteries in humans. In: Angelini, P. (ed.) Coronary artery anomalies. A comprehensive approach. Philadelphia, Lippincott Williams \& Wilkins, 1999. pp. 279.

Banchi, A. Morfologia delle arteriae coronariae cordis. Arch. Ital. Anat. Embriol., 3:87-95, 1904.

Baptista, C. A. C. \& DiDio, L. J. A. The relationship between the directions of myocardial bridges and of the branches of the coronary arteries in the human heart. Surg. Radiol. Anat., 14:137-40, 1992.

Baptista, C. A.; DiDio, L. J. \& Prates, J. C. Types of division of the left coronary artery and the ramus diagonalis of the human heart. Jpn. Heart. J., 32(3):323-35, 1991.

Bezerra, A. J. C.; Prates, J. C. \& DiDio, L. J. A. Incidence and clinical significance of bridges myocardium over the coronary arteries and their branches. Surg. Radiol. Anat., 9:273-80, 1987.

Campbell, J. S. Stereoscopic radiography of the coronary system. Q. J. Med., 22:247-67, 1929.

Canyigit, M.; Hazirolan,T.; Karcaaltincaba, M.; Dagoglu, M.
G.; Akata, D.; Aytemir, K.; Oto, A.; Balkanci, F.; Akpinar, E. \& Besim, A. Myocardial bridging as evaluated by 16 row MDCT. Eur. J. Radiol., 69:156-64, 2009.

Cavalcanti, J. S.; de Lucena Oliveira, M.; Pais e Melo, A. V.; Balaban, G.; de Andrade Oliveira, C. L. \& de Lucena Oliveira E. Anatomic variations of the coronary arteries. Arq. Bras. Cardiol., 65(6):489-92,1995.

Çay, S.; Öztürk, S.; Cihan, G.; Kisacik, H. L. \& Korkmaz, S. Angiographic prevalence of myocardial bridging. Anadolu Kardiyol. Derg., 6:9-12, 2006.

De Paula, W. Estudo estadistico sobre a irrigação coronariana no coração humano em brancos e negros. Folha. Cli. Biol., 1:20-42, 1972.

DiDio, L. J. \& Wakefield, T. W. Coronary arterial predominance or balance on the surface of the human cardiac ventricles. Anat. Anz., 137(1-2):147-58, 1975.

Duygu, H.; Zoghi, M.; Nalbantgil, S.; Kirilmaz, B., Turk, U., Ozerkan, F.; Akilli, A, \& Akin, M. Myocardial bridge: A bridge to atherosclerosis. Anadolu Kardiyol. Derg., 7:12-6, 2007.

Gow, R. M. Myocardial bridging: does it cause sudden death? Card. Electrophysiol. Rev., 6:112-4, 2002.

Grande, N.; Castelo-Branco, N. \& Ribeiro, A. Coronary arterial circulation in the Bantu. Ohio J. Sci., 82:146-52, 1982.

James, T. N. Anatomy of the coronary arteries. New York, Hoeber, 1961. 
James, T. N. Anatomy of the coronary arteries in health and disease. Circulation, 32:1020-33, 1965.

Kalbfleisch, H.; Hort, W. \& Müller E. Comparative coronarangiographic and histologic study on posterior myocardial infarction. Z. Kardiol., 66:676-84, 1977.

Kalpana, R. A study on principal branches of coronary arteries in humans. J. Anat. Soc. India, 52:137-40, 2003.

Kantarci, M.; Duran, C.; Durur, I.; Alper, F.; Onbas, O.; Gulbaran, M. \& Okur, A. Detection of myocardial bridging with ECGgated MDCT and multiplanar reconstruction. Am. J. Roentgenol., 186:S391-4, 2006.

Ko, S. M.; Choi, J. S.; Nam, C. W. \& Hur, S. H. Incidence and clinical significance of myocardial bridging with ECG-gated 16-row MDCT coronary angiography. Int. J .Cardiovasc. Imaging., 24:445-52, 2008.

Kosinski, A. \& Grzybiak, M. Myocardial bridges in the human heart: morphological aspects. Folia Morphol. (Warsz), 60(1):65-8, 2001.

Leguerrier, A.; Calmat, A.; Honnart, F. \& Cabrol, C. Anatomic variations of the common trunk of the left coronary artery (apropos of 80 dissections). Bull. Assoc. Anat. (Nancy), 60:733-9, 1976.

Lima, V. J.; Cavalcanti, J. S. \& Tashiro, T. Myocardial bridges and their ralationship to the anterior interventricular branch of the left coronary artery. Arq. Bras. Cardiol., 79:219-22, 2002.

Loukas, M.; Curry, B.; Bowers, M.; Louis, Jr., R. G.; Bartczak, A.; Kiedrowski, M.; Kamionek, M.; Fudalej, M. \& Wagner, T. The relationship of myocardial bridges to coronary artery dominance in the adult human. J. Anat., 209: 43-50, 2006.

Margaris, N. G.; Kostopoulos, K. G.; Nerantzis, C. E.; Filippatos, G. S.; Kardaras, F. G.; Salahas, A. I.; Antonellis, J. P.; Ifandis, G. P.; Kranidis, A. I. \& Tavernarakis, A. G. Posterior right diagonal artery. An angiographic study. Angiology., 48(8):673-7, 1997.

McAlpine, W. A. Heart and coronary arteries: An anatomical atlas for clinical diagnosis, radiological investigation and surgical treatment. Berlin, Springer-Verlag, 1975. pp. 163209.

Nerantzis, C. E.; Antonakis, E. \& Avgoustakis, D . A new corrosion casting technique. Anat. Rec., 191:321-6, 1978.

Nerantzis, C. E.; Gribizi, J. E.; Margaris, N. G.; Antonelis, J. P.; Salahas, T. I. \& Koroxenidis, G. T. Posterior right diagonal artery. Anat. Rec., 238:528-32, 1994.
Ortale, J. R.; Keiralla, L. C. \& Sacilotto, L. The posterior ventricular branches of the coronary arteries in the human heart. Arq. Bras. Cardiol., 82(5):468-72, 2004.

Ortale, J. R.; Meciano Filho, J.; Paccola A. M. F.; Leal, J. G. P. G. \& Scaranari, C. A. Anatomia dos ramos lateral, diagonal e ântero-superior no ventrículo esquerdo do coração humano. Rev. Bras. Cir Cardiovasc., 20(2):149-58, 2005.

Penthe, P.; Bara, J. A. \& Blanc, J. J. Etude anatomique descriptive des gros troncs coronariens et des principales collaterales epicardiques. Nouv. Presse. Med., 5:71-5, 1976.

Polacek, P. Relation of myocardial bridges and loops on the coronary arteries to coronary occlusions. Am. Heart J., 61:44$52,1961$.

Quagliano, A. P.; Cury, L.; Pereira, R. A. A. \& Prates N. E. Puentes de miocardio sobre las arterias coronarias y su importancia práctica. Archivos Anatómicos de Costa Rica, 1:137-8, 1992.

Reig, J.; Loncan, M. P.; Martin, S.; Binia, M.; Petit, M. \& Domenech, J. M. Myocardial bridges. Incidence and relation to some certain coronary variables. Arch. Anat. Histol. Embryol., 69:101-10, 1986.

Reig, J. \& Petit, M. Main trunk of the left coronary artery: Anatomic study of the parameters of clinical interest. Clin. Anat., 17(1):6-13, 2004.

Rozenberg, V. D. \& Nepomnyashchikh, L. M. Pathomorphology and pathogenic role and myocardial bridges in sudden cardiac death. Bull. Exp. Biol. Med., 138:87-92, 2004

Sahni, D. \& Jit, I. Incidence of myocardial bridges in Northwest Indians. Indian Heart J., 43(6):431-6, 1991.

Sewell, W. H. Roentgenographic anatomy of human coronary arteries. Am. J. Roentgenol. Radium. Ther. Nucl. Med., 97:35968, 1966.

Schlesinger, M. J. Relation of anatomic pattern to pathologic conditions of the coronary arteries. Arch. Pathol., 30:40315,1940 .

Smith, G. T. The anatomy of the coronary circulation. Am. J. Cardiol., 9:327-42, 1962.

\section{Correspondence to:}

Prof. Dr. Luis Ernesto Ballesteros

Departamento Ciencias Básicas.

Universidad Industrial de Santander.

Bucaramanga

COLOMBIA.

Email: Iballest56@yhoo.es

Received: 19-06-2009

Accepted: 27-08-2009 